\title{
LncRNA RUNXI-ITI is Downregulated in Endometrial Cancer and Binds to miR-2I Precursor to Suppress Its Maturation
}

This article was published in the following Dove Press journal: Cancer Management and Research

\section{Minglin Liang Hongbo Wang Cong Liu \\ Tao Lei \\ Jie Min}

Department of Obstetrics and Gynecology, Union Hospital Affiliated to Tongji Medical College of Huazhong University of Science and Technology, Wuhan City, Hubei Province 430022 People's Republic of China
Correspondence: Jie Min

Department of Obstetrics and Gynecology, Union Hospital Affiliated to Tongji Medical College of Huazhong University of Science and Technology, I 277 Jiefang Avenue, Wuhan, Hubei Province, People's Republic of China Email minjieobstetrics@I63.com
Background: RUNX1-IT1 suppresses colorectal cancer and liver cancer, while its role in other cancers is unknown. This study was performed to investigate the role of RUNX1-IT1 in endometrial cancer (EC).

Methods: EC and paired non-tumor tissues were collected from $62 \mathrm{EC}$ patients, and the expression of RUNX1-IT1, mature miR-21 and miR-21 precursor in these tissue samples were determined by RT-qPCR. Correlations were analyzed by linear regression. Overexpression of RUNX1-IT1 was achieved in EC cells and the expression of mature miR-21 and miR-21 precursor were analyzed by RT-qPCR. CCK- 8 assay was used for cell proliferation analysis.

Results: We found that RUNX1-IT1 was downregulated in EC and inversely correlated with mature miR-21 but not miR-21 precursor. RUNX1-IT1 was predicted to bind with miR-21 precursor. The interaction between them was verified by dual-luciferase activity assay and RNA pull-down assay. In EC cells, overexpression of RUNX1-IT1 downregulated mature miR-21, but not miR-21 precursor. Overexpression of RUNX1-IT1 suppressed the role of miR-21 in increasing cell proliferation.

Conclusion: RUNX1-IT1 is downregulated in EC and inhibits cancer cell proliferation by suppressing the maturation of miR-21.

Keywords: endometrial cancer, RUNX1-IT1, miR-21, precursor, proliferation

\section{Introduction}

Endometrial cancer is a type of malignancy that originates from endometrium and it mainly affects females older than 55 years. ${ }^{1}$ In developing countries, EC is the most common type of gynecologic tumors with an increasing incidence. ${ }^{2}$ EC patients in China account for about $12 \%$ of all EC cases. ${ }^{3}$ It is estimated that more than $95 \%$ of patients with localized EC can survive 5 years. ${ }^{4}$ However, metastases to distant sites such as the bladder, rectum, ovary, and vagina are common in EC patients. ${ }^{5}$ Due to the lack of effective treatment strategies, the 5-year overall survival rate of patients with distant metastatic EC is below 20\%. ${ }^{6}$ Therefore, novel therapies are needed to further improve patients' survival.

Studies have characterized various molecular alterations involved in the initiation, development and progression of EC. ${ }^{7,8}$ Some molecular pathways, such as the EMP2 and Wnt signaling pathways, are potential targets for the development of novel targeted therapies, which can be utilized to regulate cancer-related network of gene expression to suppress cancer. ${ }^{9,10}$ However, effective targets for the treatment of EC remain lack. 
MicroRNAs (miRNAs) and long non-coding RNAs (lncRNAs) are two types of ncRNAs that are not proteincoding transcripts but participate in human diseases by regulating related gene expression. ${ }^{11}$ Therefore, miRNAs and IncRNAs are promising targets for targeted therapies. LncRNA RUNX1-IT1 has been characterized as a tumor suppressor in liver cancer and colorectal cancer. ${ }^{12-14}$ However, its role in other cancers is unknown. We predicted that RUNX1IT1 might interact with miR-21, which is an important player in cancer biology. ${ }^{15}$ We therefore explored the potential interaction between RUNX1-IT1 and miR-21 in EC.

\section{Patients and Methods}

\section{EC Patients}

This study enrolled a total of 62 EC patients admitted at Union Hospital affiliated to Tongji Medical College of Huazhong University of Science and Technology from June 2018 to April 2020. The age of patients ranged from 49 to 68 years old (mean age $58.7 \pm 5.7$ years old). Patients complicated with other clinical disorders or with initiated therapies were excluded from this study. All the $62 \mathrm{EC}$ patients were excluded from recurrent cases. Based on the system of AJCC, the $62 \mathrm{EC}$ patients were classified into stage I or II $(n=30)$, and III or IV $(n=32)$. All patients signed the written informed consent. This study was approved by the Ethics Committee of aforementioned Hospital. The work described has been carried out in accordance with The Code of Ethics of the World Medical Association (Declaration of Helsinki) for experiments involving humans.

\section{EC Tissues and Cells}

Prior to therapy, EC and paired non-tumor tissues were collected through fine needle aspiration. Histopathological exam was performed on all tissue samples to confirm that correct tissue samples were obtained. RNA isolation was performed immediately after confirmation.

Two EC cell lines RL95-2 (carcinoma) and HEC1-A (adenocarcinoma) obtained from ATCC (USA) were used as in vitro cell model of EC. DMEM (Sigma-Aldrich) supplemented with 10\% FBS (PAA Laboratories) was used as cell culture medium. Cells were cultivated in an incubator at $37^{\circ} \mathrm{C}$ with $95 \%$ humidity and $5 \% \mathrm{CO}_{2}$.

\section{Transient Transfections and}

\section{Dual-Luciferase Reporter Assay}

With pcDNA3.1 vector (Invitrogen) as the backbone, vector expressing RUNX1-IT1 was established. Mimic of
miR-21 and negative control (NC) mimic were synthesized by Sigma-Aldrich. Through lipofectamine 2000 (Invitrogen)-mediated transient transfections, RL95-2 and HEC-1-A cells $\left(10^{7}\right)$ were transfected with $1 \mu \mathrm{g}$ RUNX1IT1 expression vector or $45 \mathrm{nM}$ miRNA. To perform NC experiments, the same amount of empty vector or $\mathrm{NC}$ miRNA was transfected into the same number of cells. Prior to subsequent experiments, cells were cultivated in fresh medium for $48 \mathrm{~h}$ after transfections.

With pGL3 luciferase reporter vector (Promega Corporation) as the backbone, RUNX1-IT1 luciferase vector was constructed. Briefly, the binding site of miR-21 on RUNX1-IT1 was inserted into the region between SV40 promoter and Luc $(+)$. Cells were transfected with RUNX1IT1 luciferase vector combined with NC miRNA precursor (NC group) or miR-21 precursor (miR-21 group). Luciferase activity was determined at $48 \mathrm{~h}$ post-transfection. After transcription, a fusion RNA composed of RUNX1-IT1 + Luc (+) was produced. The binding of miR-21 to the fusion RNA reduced the translation of Luc $(+)$, resulting in decreased luciferase signal. ${ }^{16,17}$

\section{RNA Pull-Down Assay}

Biotinylated RUNX1-IT1 RNA (Bio-RUNX1-IT1) and NC (Bio-NC) were purchased from Invitrogen (Shanghai, China). RL95-2 and HEC-1-A cells were transfected with Bio-RUNX1-IT1 or Bio-NC. At $48 \mathrm{~h}$ post-transfection, cells were lysed and incubated with streptavidin magnetic beads (Invitrogen) to isolate Bio-RUNX1-IT1 and NC (Bio-NC). Finally, qPCRs were performed to determine the expression levels of miR-21 in these samples.

\section{RNA Preparations}

Ribozol (Invitrogen) was used to isolate total RNAs from paired tissues and in vitro cultivated cells. RNA samples were incubated with DNase I (Invitrogen) at $37^{\circ} \mathrm{C}$ for 100 min to completely remove genomic DNA. A 5\% ureaPAGE gel was used to separate RNAs to check RNA integrity. RNA purity was reflected by OD 260/280 ratios.

\section{RT-qPCR}

RNA samples with OD ratios closely to 2.0 (pure RNA samples) were used for the preparation of cDNA samples using the SS-IV-RT system (Invitrogen). The qPCR reaction systems were prepared using SYBR Green Master Mix (BioRad, USA). The expression levels of RUNX1-IT1 were determined using 18S rRNA as the internal control. The expression levels of miR-21 precursor and mature miR-21 
were determined using Genecopoeia All-in-One ${ }^{\mathrm{TM}}$ miRNA qRT-PCR Detection Kit. Sequence-specific forward and reverse primers were used to determine the expression of miR-21 precursor. To determine the expression of mature miR-21, poly (A) was added to mature miRNAs, and poly (T) reverse primer and sequence-specific forward primer were used to perform reverse transcription and qPCRs. Three technical replicates were included in each qPCR. Ct values of targeted genes were normalized to internal controls using $2^{-\Delta \Delta C T}$ method.

\section{Cell Counting Kit-8 (CCK-8) Assay}

The proliferation of RL95-2 and HEC-1-A cells was determined by CCK-8 assay at $48 \mathrm{~h}$ post-transfection. A 96well cell culture plate was used to cultivate cells with 3000 cells per well at $37^{\circ} \mathrm{C}$. To determine cell proliferation, OD values $(450 \mathrm{~nm})$ were determined every $24 \mathrm{~h}$ for a total of
$4 \mathrm{~d}$. Each well was added with $10 \%$ CCK-8 solution at 2 $\mathrm{h}$ before the measurement of OD values.

\section{Transwell Assay}

Cell migration and invasion abilities were measured by transwell assay using 24-Transwell plates with the $8.0 \mu \mathrm{m}$ pore size membrane (Corning Incorporated). Transfected cells were seeded onto the upper chamber of 24-Transwell plates, $700 \mu \mathrm{L}$ of growth medium containing 10\% FBS was added into the lower chamber as chemoattractant. After $24 \mathrm{~h}$ of incubation, the migrated or invaded cells were stained with $0.1 \%$ crystal violet. The number of migrated or invaded cells was counted under a Nikon microscope (Nikon, Japan).

\section{Flow Cytometry}

Flow cytometry was used to determine cell apoptosis using the Annexin V-FITC/PI Apoptosis Detection Kit (Invitrogen).
A

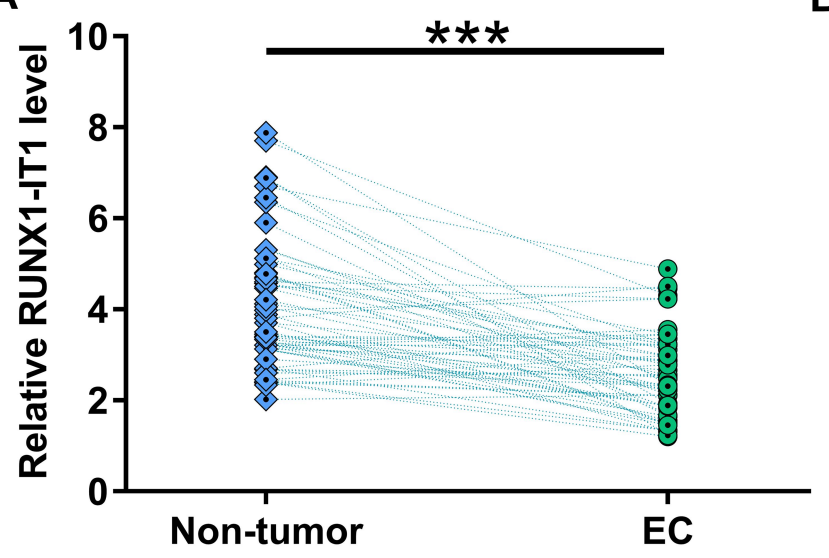

C

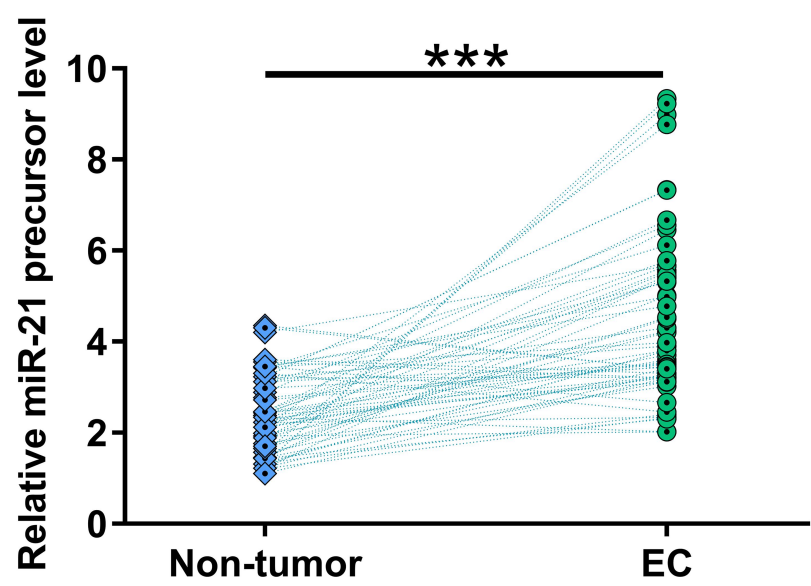

B

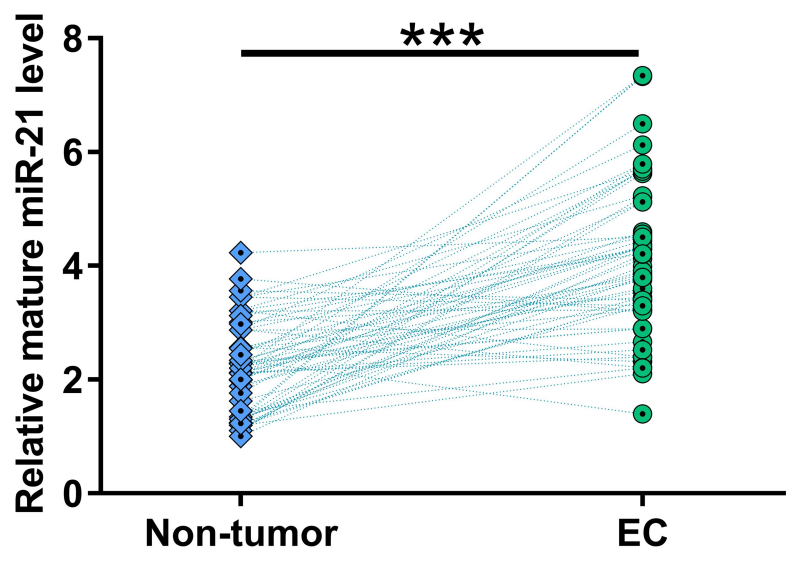

Figure I Altered expression of RUNXI-ITI, mature miR-2 and miR-2I precursor were observed in EC. The 62 pairs of EC and non-tumor tissues were subjected to RNA isolations and RT-qPCRs to determine the expression of RUNXI-ITI (A), mature miR-2I (B) and miR-2I precursor (C). Gene expression levels in tissue samples were expressed as mean values of three technical replicates. $*^{* * *} p<0.00 \mathrm{I}$. 
Table I Chi-Squared Test Analysis of the Correlation Between RUNXI-ITI Expression and Patients' Clinical Data

\begin{tabular}{|c|c|c|c|c|c|c|}
\hline Items & Groups & Cases & High-Expression & Low-Expression & $\chi^{2}$ & $p$ value \\
\hline \multirow[t]{2}{*}{ Age } & > 60 (years old) & 26 & 12 & 14 & \multirow[t]{2}{*}{0.56} & \multirow[t]{2}{*}{0.45} \\
\hline & $<60$ (years old) & 36 & 19 & 15 & & \\
\hline \multirow[t]{2}{*}{$\mathrm{AJCC}$} & I or II & 30 & 13 & 17 & \multirow[t]{2}{*}{1.03} & \multirow[t]{2}{*}{0.31} \\
\hline & III or IV & 32 & 18 & 14 & & \\
\hline \multirow[t]{2}{*}{ Multiplicity } & Single & 20 & 12 & 8 & \multirow[t]{2}{*}{0.18} & \multirow[t]{2}{*}{0.28} \\
\hline & Multiple & 42 & 19 & 23 & & \\
\hline \multirow[t]{2}{*}{ Subtype } & Carcinoma & 28 & 15 & 13 & \multirow[t]{2}{*}{0.26} & \multirow[t]{2}{*}{0.61} \\
\hline & Adenocarcinoma & 34 & 16 & 18 & & \\
\hline \multirow[t]{2}{*}{ History of endometrial hyperplasia } & Yes & 12 & 7 & 5 & \multirow[t]{2}{*}{$0.4 I$} & \multirow[t]{2}{*}{0.52} \\
\hline & No & 50 & 24 & 26 & & \\
\hline \multirow[t]{2}{*}{ Diabetes } & Yes & 18 & 10 & 8 & \multirow[t]{2}{*}{0.31} & \multirow[t]{2}{*}{0.58} \\
\hline & No & 44 & 21 & 23 & & \\
\hline \multirow[t]{2}{*}{ Obesity } & Yes & 16 & 7 & 9 & \multirow[t]{2}{*}{0.34} & \multirow[t]{2}{*}{0.56} \\
\hline & No & 46 & 24 & 22 & & \\
\hline
\end{tabular}

Transfected cells were harvested and resuspended in Binding Buffer $(1 \times)$, then incubated with Annexin VPITC and PI at $37^{\circ} \mathrm{C}$ for $30 \mathrm{~min}$ in the dark. The apoptotic rate was analyzed by FACScan flow cytometer (Becton Dickinson) using the CELL QUEST software.

\section{Statistical Analyses}

Gene expression levels in tissue samples were expressed as the mean values of three technical replicates and were compared by paired $t$ test. Mean \pm stand deviation (SD) values of three independent replicates were used to express data of multiple transfection groups. Comparison between two groups was performed by unpaired $t$ test, and comparisons among multiple groups were performed by ANOVA Tukey's test. The median expression level of RUNX1-IT1 in EC tissues was used as a cutoff value to divide the 62 EC patients into high and low RUNX1-IT1 level groups. Correlations between the expression levels of RUNX1-IT1 and patients' clinical data were analyzed by Chi-squared test. $p<0.05$ was considered as statistically significant.
A

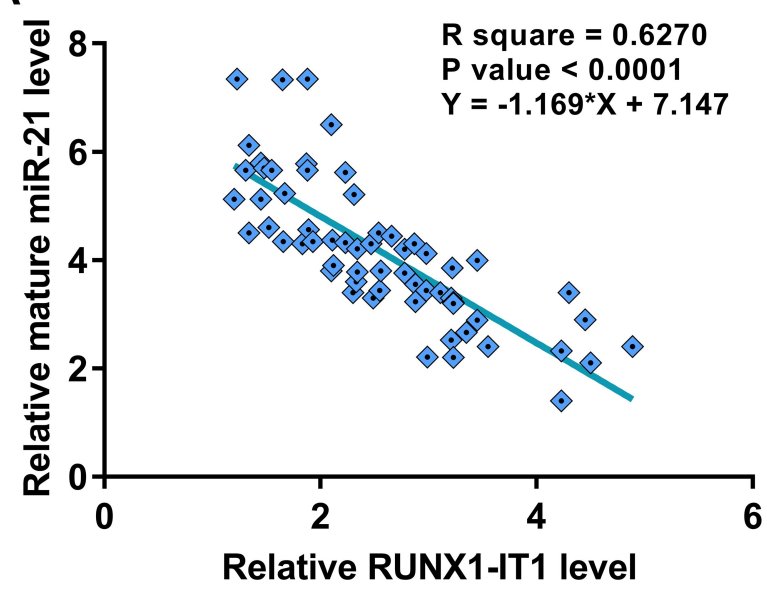

B

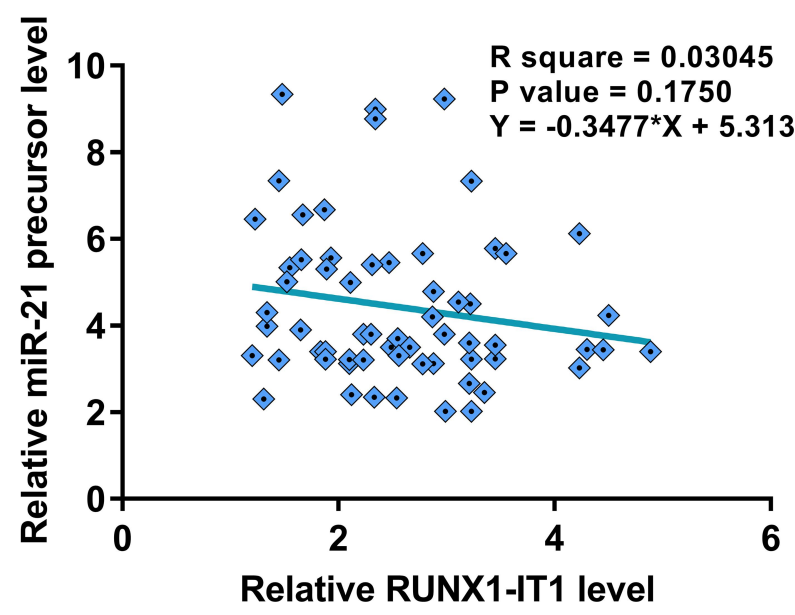

Figure 2 RUNXI-ITI and mature miR-2I were inversely correlated across EC tissues. Linear regression was performed to analyze the correlations between RUNXI-ITI and mature miR-2I (A) or miR-2I precursor $(\mathbf{B})$ across EC tissues. 


\section{Results}

\section{Altered Expression of RUNXI-ITI,} Mature miR-2I and miR-2I Precursor Were Observed in EC

RT-qPCRs results showed that RUNX1-IT1 was significantly downregulated in EC tissues (Figure 1A, $p<0.001$ ). In contrast, the expression levels of mature miR-21 (Figure $1 \mathrm{~B}, p<0.001$ ) and miR-21 precursor (Figure 1C, $p<0.001)$ were significantly higher in EC tissues. Chisquared test analysis showed that the expression of RUNX1-IT1 was not significantly correlated with patients' age, clinical stage, tumor multiplicity, history of endometrial hyperplasia, diabetes and obesity (Table 1).

\section{RUNXI-ITI and Mature miR-2I Were Inversely Correlated Across EC Tissues}

Correlations between RUNX1-IT1 and mature miR-21 or miR-21 precursor across EC tissues were explored by linear regression. It was observed that RUNX1-IT1 was inversely correlated with mature miR-21 across EC tissues (Figure 2A). In contrast, RUNX1-IT1 and miR-21 precursor were not significantly correlated with each other across EC tissues (Figure 2B).

\section{RUNXI-ITI Directly Interacted with miR-2I}

The interaction between RUNX1-IT1 and miR-21 precursor was predicted by IntaRNA 2.0 (http://rna.informatik.unifreiburg.de/IntaRNA/Input.jsp). It showed that RUNX1-IT1 and miR-21 precursor may form strong base pairing (Figure 3A). Dual-luciferase reporter assay showed that the miR-21 group exhibited significantly lower level of luciferase activity compared to that in the $\mathrm{NC}$ group, suggesting direct interaction between them (Figure 3B, $p<0.05$ ). RNA-pull down assay was performed to further explore the interaction between them. Compared to the Bio-NC group, BioRUNX1-IT1 group showed significantly higher expression

A

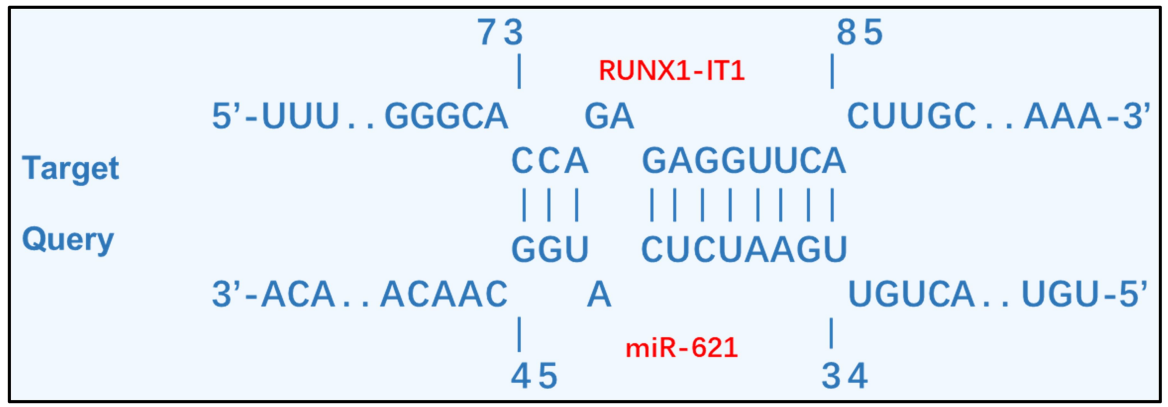

B

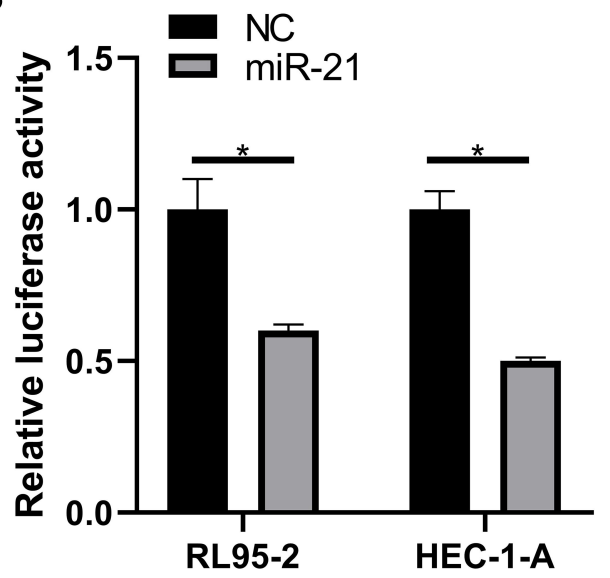

C

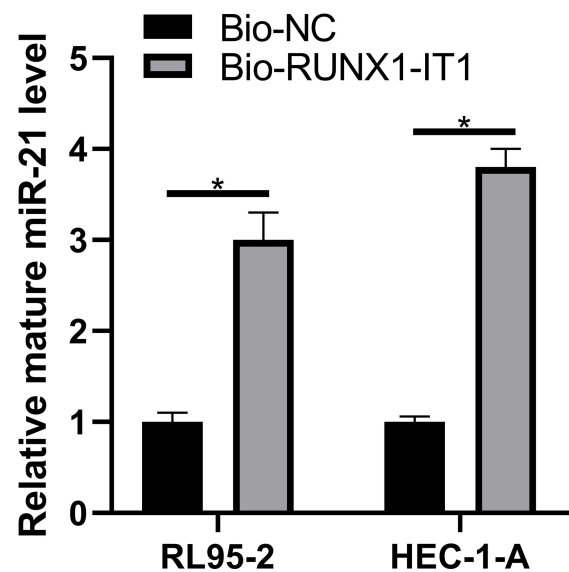

Figure 3 RUNXI-ITI could directly interact with miR-2I. The interaction between RUNXI-ITI and miR-2I precursor was predicted by IntaRNA 2.0 (A). Dual-luciferase reporter assay was performed by transfecting RUNXI-ITI luciferase vector combined with NC miRNA precursor (NC group) or miR-2I precursor sequence into RL95-2 and HEC-I-A cells, followed by the determination of luciferase activity at $48 \mathrm{~h}$ post-transfection (B). RNA pull-down assay was performed to use Bio-RUNXI-ITI and BioNC to pull down miR-2I. qPCR was used to determine the expression of mature miR-2I in each pull-down groups $(\mathbf{C})$. ${ }^{*} p<0.05$. 

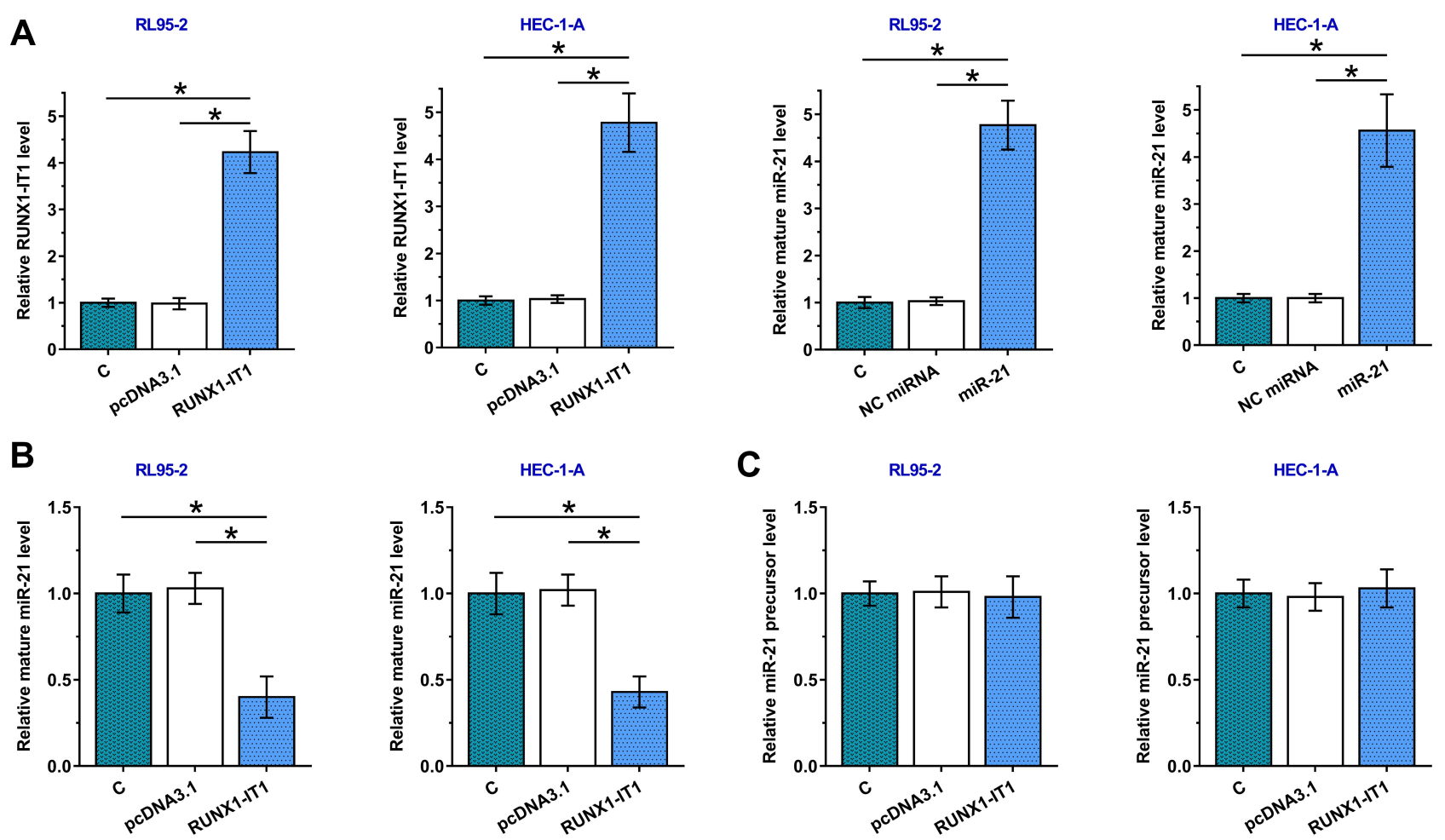

D
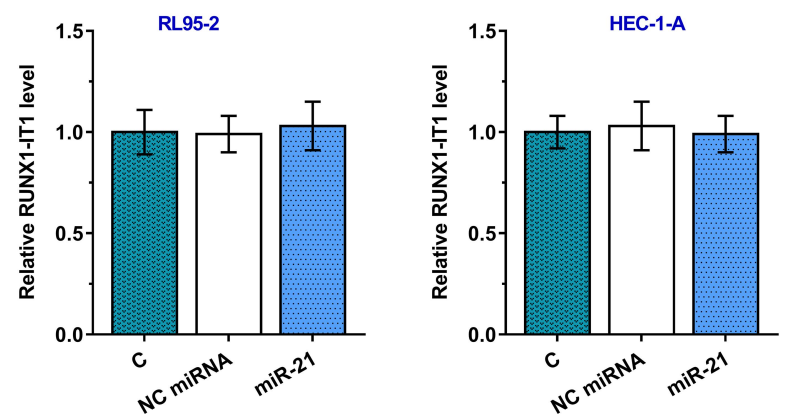

Figure 4 RUNXI-ITI suppressed the maturation of miR-2I. RL95-2 and HEC-I-A cells were transfected with either RUNXI-ITI expression vector or miR-2I mimic, followed by the confirmation of the overexpression of RUNXI-ITI and miR-2I by RT-qPCR (A). The effects of overexpression of RUNXI-ITI on mature miR-2I (B) and miR-2I precursor (C), and the effects of overexpression of miR-2I on RUNXI-ITI (D) were analyzed by RT-qPCR. Mean \pm SD values of three independent replicates were used to express data of multiple transfection groups. ${ }^{*} p<0.05$.

levels of mature miR-21, further confirming the interaction between RUNX1-IT1 and miR-21 (Figure 3C, $p<0.05$ ).

\section{RUNXI-ITI Suppressed the Maturation of miR-2I}

To explore the interaction between RUNX1-IT1 and miR21, RL95-2 and HEC-1-A cells were transfected with either RUNX1-IT1 expression vector or miR-21 mimic, followed by the confirmation of the overexpression of RUNX1-IT1 and miR-21 by RT-qPCR (Figure 4A, $p<$ 0.05). It was observed that overexpression of RUNX1-IT1 resulted in significantly downregulated mature miR-21 (Figure 4B, $p<0.05$ ), but did not alter the expression of
miR-21 precursor (Figure 4C). In addition, the expression of RUNX1-IT1 was not altered after the overexpression of miR-21 (Figure 4D).

\section{Overexpression of RUNXI-ITI Resulted in Decreased Proliferation of EC Cells}

\section{Through miR-2I}

The effect of RUNX1-IT1 and miR-21 on RL95-2 and HEC-1-A cell proliferation was analyzed by CCK-8 assay. Overexpression of RUNX1-IT1 decreased the proliferation of EC cells, while overexpression of miR-21 played an opposite role. In addition, overexpression of 


\section{RL95-2}

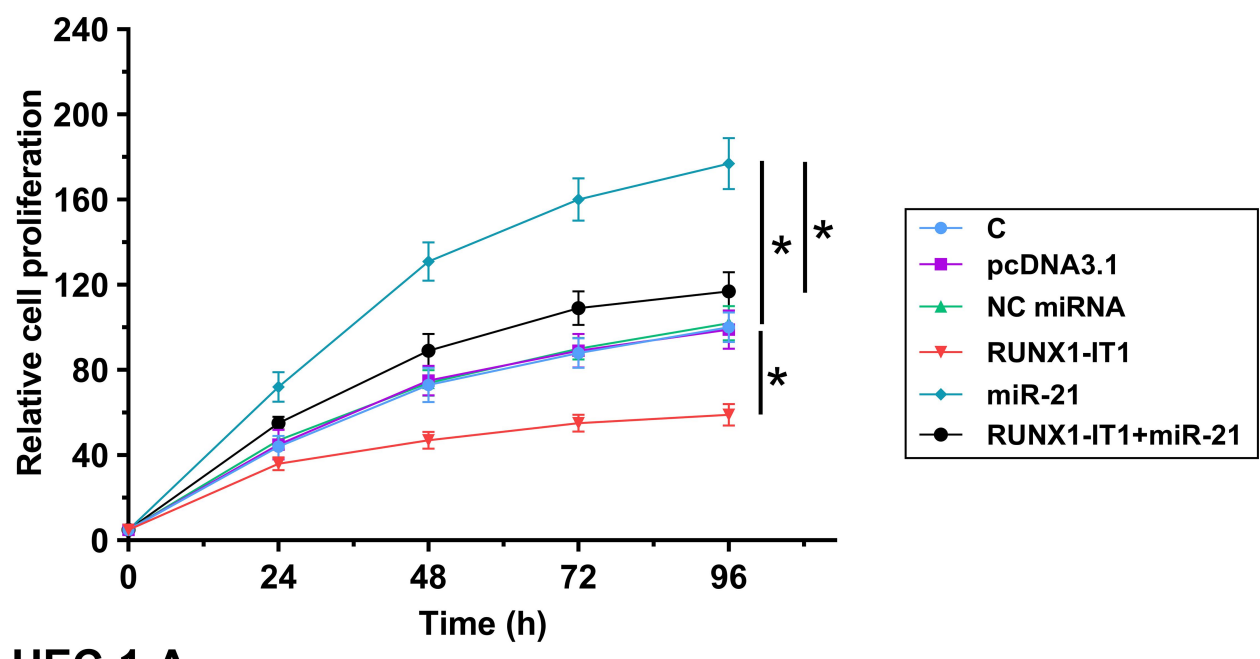

\section{HEC-1-A}

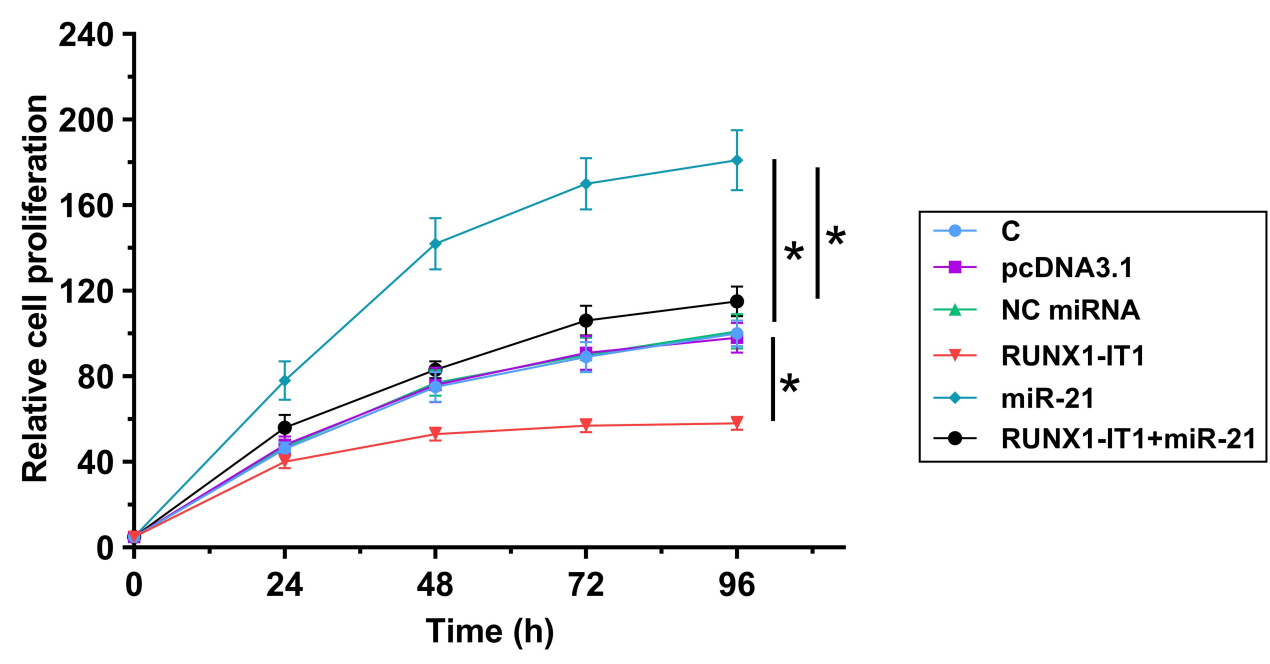

Figure 5 Overexpression of RUNXI-ITI resulted in decreased proliferation of EC cells through miR-2I. The roles of RUNXI-ITI and miR-2I in regulating the proliferation of RL95-2 and HEC-I-A cells were analyzed by CCK-8 assay. Mean \pm SD values of three independent replicates were used to express data of multiple transfection groups. $*_{p}<0.05$.

RUNX1-IT1 attenuated the role of overexpression of miR21 in promoting cell proliferation (Figure 5, $p<0.05$ ). Moreover, Transwell assay and cell apoptosis assay data showed that overexpression of RUNX1-IT1 did not affect the invasion, migration and apoptosis of RL95-2 and HEC-1-A cells (Figure 6).

\section{Discussion}

This study analyzed the expression of RUNX1-IT1 in EC and explored its crosstalk with miR-21. We found that RUNX1IT1 was downregulated in EC and it suppressed the maturation of miR-21 in EC cells to suppress cancer cell proliferation.

RUNX1-IT1 suppresses colorectal cancer and liver cancer. ${ }^{12-14}$ RUNX1-IT1 is downregulated in colorectal cancer, and overexpression of RUNX1-IT1 plays a tumor suppressive role by inhibiting cancer cell migration and proliferation, while the mechanism of the function of RUNX1IT1 in this disease remains unclear. In liver cancer, RUNX1IT1 interacts with the MAPK pathway to suppress cell proliferation and apoptosis. ${ }^{13}$ In addition, hypoxia-driven histone deacetylase 3 is characterized as a suppressor of RUNX1IT1 in liver cancer cells. ${ }^{14}$ In this study, RUNX1-IT1 was shown to be downregulated in EC. Moreover, overexpression of RUNX1-IT1 resulted in decreased proliferation of EC cells. Therefore, RUNX1-IT1 plays a tumor suppressive role in EC and overexpression of RUNX1-IT1 has the potential to be targeted to treat EC. However, in vivo experiments and clinical studies are still needed to further confirm our conclusions.

MiR-21 is a master player in cancer biology. ${ }^{15,18}$ MiR-21 targets PTEN to suppress the apoptosis of cancer cells and 

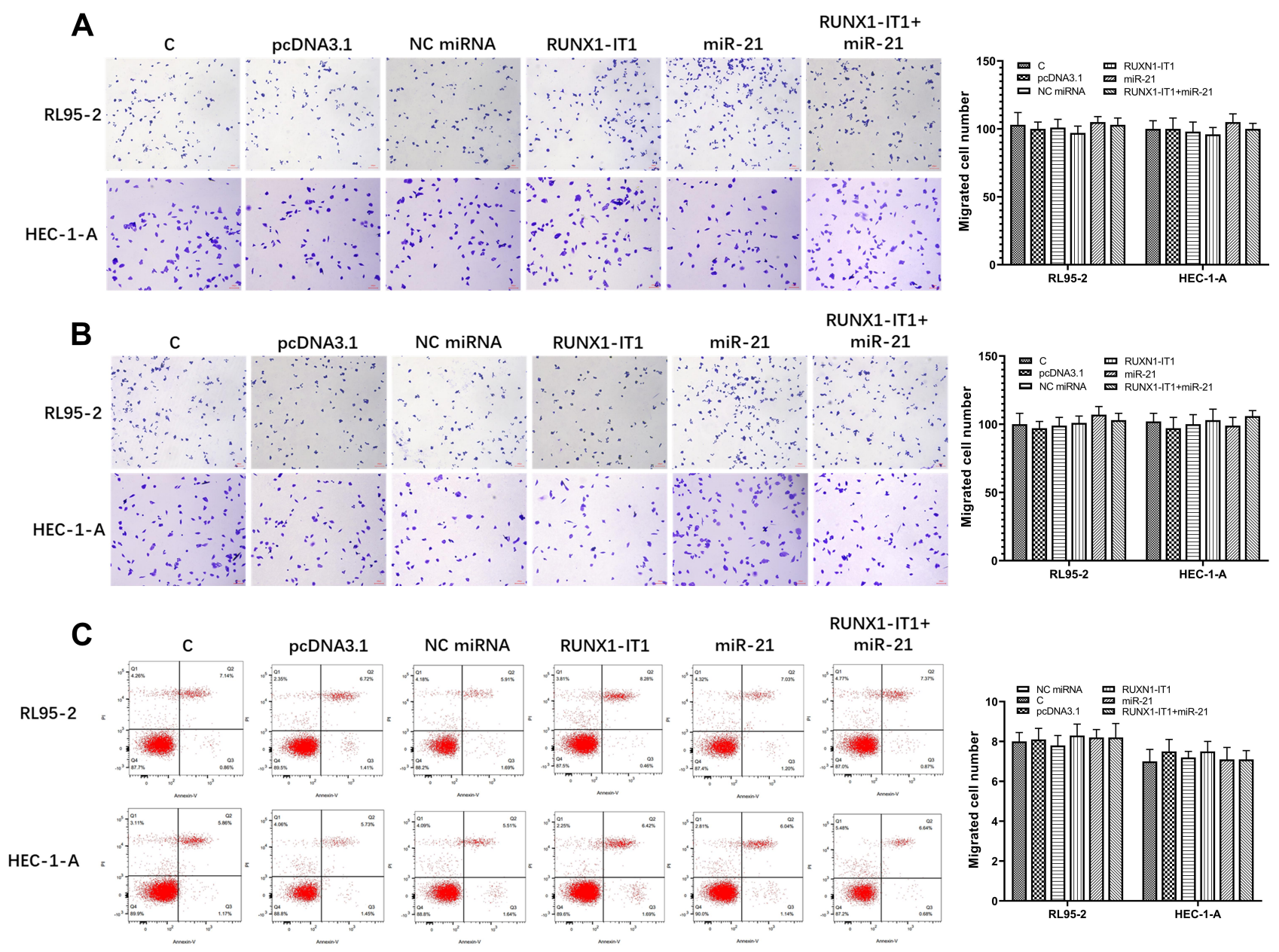

Figure 6 Overexpression of RUNXI-ITI did not affect the invasion, migration and apoptosis of EC cells. Overexpression of RUNXI-ITI did not significantly affect the invasion (A), migration (B) and apoptosis (C) of RL95-2 and HEC-I-A cells.

promotes cell proliferation. ${ }^{15}$ In addition, overexpression of miR-21 also induces the development of chemoresistance during chemotherapy, resulting in the failures of treatment. ${ }^{18}$ Therefore, inhibition of the expression of miR-21 is considered as a promising target for cancer treatment. In this study, we confirmed the oncogenic role of miR-21 in EC. Interestingly, we provided evidence that RUNX1-IT1 may suppress the maturation of miR-21 from precursor to mature miRNA in EC cells. Although we did not include in vivo experiment, we observed the inversely correlation between RUNX1-IT1 and mature miR-21, but not RUNX1-IT1 and miR-21 precursor. Therefore, RUNX1-IT1 may also suppress the maturation of miR-21 in human body. However, the mechanism is unclear. A recent study proposed that IncRNA CCAT2 may suppress the maturation of miR-145 by inhibiting its transportation from nucleus to cytoplasm. ${ }^{19}$ Therefore, RUNX1-IT1 may also suppress the movement of miR-21. RL95-2 is a carcinoma cell line and HEC-1-A is an adenocarcinoma cell line. It is noteworthy that no significant differences in cellular assay data were observed between RL95-2 and HEC-1-A cells. Therefore, RUNX1-IT1 may play similar roles in two different subtypes of EC.

\section{Conclusions}

In conclusion, RUNX1-IT1 is downregulated in EC and it may suppress the maturation of miR-21 to inhibit EC cell proliferation.

\section{Ethics Approval and Consent to Participate}

Ethical approval was obtained from the Ethics Committee of Union Hospital affiliated to Tongji Medical College of Huazhong University of Science and Technology. The work described has been carried out in accordance with The Code of Ethics of the World Medical Association 
(Declaration of Helsinki) for experiments involving humans. Informed consent was obtained from the all the patients.

\section{Disclosure}

The authors declare that they have no competing interests.

\section{References}

1. Siegel RL, Miller KD, Jemal A. Cancer statistics, 2019. CA Cancer J Clin. 2019;69(1):7-34.

2. Morice P, Leary A, Creutzberg C, Abu-Rustum N, Darai E. Endometrial cancer. Lancet. 2016;387(10023):1094-1108.

3. Zhang Y, Liu Z, Yu X, et al. The association between metabolic abnormality and endometrial cancer: a large case-control study in China. Gynecol Oncol. 2010;117:41-46.

4. Sanni O, Mc Menamin Ú, Cardwell C, Sharp L, Murray L, Coleman H. Commonly used medications and endometrial cancer survival: a population-based cohort study. $\mathrm{Br} J$ Cancer. 2017;117:432-438.

5. Liu Y, Chi S, Zhou X, Zhao R, Xiao C, Wang H. Prognostic value of distant metastatic sites in stage IV endometrial cancer: a SEER database study of 2948 women. Int J Gynaecol Obstet. 2020;149(1):16-23.

6. Beavis AL, Yen -T-T, Stone RL, et al. Adjuvant therapy for early stage, endometrial cancer with lymphovascular space invasion: is there a role for chemotherapy? Gynecol Oncol. 2020;156(3):568-574.

7. Arend RC, Jones BA, Martinez A, Goodfellow P. Endometrial cancer: molecular markers and management of advanced stage disease. Gynecol Oncol. 2018;150(3):569-580.

8. Yen TT, Wang TL, Fader AN, Shih IM, Gaillard S. Molecular classification and emerging targeted therapy in endometrial cancer. Int J Gynecol Pathol. 2020;39(1):26-35.

9. Lheureux S, Oza AM. Endometrial cancer-targeted therapies myth or reality? Review of current targeted treatments. Eur $J$ Cancer. 2016;59:99-108.
10. Kiyohara MH, Dillard C, Tsui J, et al. EMP2 is a novel therapeutic target for endometrial cancer stem cells. Oncogene. 2017;36 (42):5793-5807.

11. Liz J, Esteller M. lncRNAs and microRNAs with a role in cancer development. Biochim Biophys Acta. 2016;1859(1):169-176.

12. Shi J, Zhong X, Song Y, et al. Long non-coding RNA RUNX1-IT1 plays a tumour-suppressive role in colorectal cancer by inhibiting cell proliferation and migration. Cell Biochem Funct. 2019;37(1):11-20.

13. Yan PH, Wang L, Chen H, et al. LncRNA RUNX1-IT1 inhibits proliferation and promotes apoptosis of hepatocellular carcinoma by regulating MAPK pathways. Eur Rev Med Pharmacol Sci. 2019;23 (19):8287-8294.

14. Sun L, Wang L, Chen T, et al. LncRNA RUNX1-IT1 which is downregulated by hypoxia-driven histone deacetylase 3 represses proliferation and cancer stem-like properties in hepatocellular carcinoma cells. Cell Death Dis. 2020;11(2):95.

15. Rak B, Marczewska JM, Wlodarski P. The role of microRNAs in endometrial cancer and influence on future therapy: focusing on miRNA-21. Eur J Gynaecol Oncol. 2016;37(5):599-603.

16. Xin D, Xin Z. CircRNA_100782 promotes roliferation and metastasis of gastric cancer by downregulating tumor suppressor gene $\mathrm{Rb}$ by adsorbing miR-574-3p in a sponge form. Eur Rev Med Pharmacol Sci. 2020;24(17):8845-8854.

17. Liang D, Wang C, Li J, Qin L, Su Z, Hou H. [Constraction of triple negative breast cancer cell model containing EGFR promoter and luciferase reporter gene]. Xi Bao Yu Fen Zi Mian Yi Xue Za Zhi= Chin J Cell Mol Immunol. 2020;36(10):918-923. Chinese.

18. Chen J, Xu T, Chen C. The critical roles of miR-21 in anti-cancer effects of curcumin. Ann Transl Med. 2015;3(21):330.

19. Yu Y, Nangia-Makker P, Farhana L, Majumdar APN. A novel mechanism of lncRNA and miRNA interaction: CCAT2 regulates miR-145 expression by suppressing its maturation process in colon cancer cells. Mol Cancer. 2017;16(1):155.
Cancer Management and Research

\section{Publish your work in this journal}

Cancer Management and Research is an international, peer-reviewed open access journal focusing on cancer research and the optimal use of preventative and integrated treatment interventions to achieve improved outcomes, enhanced survival and quality of life for the cancer patient.

\section{Dovepress}

The manuscript management system is completely online and includes a very quick and fair peer-review system, which is all easy to use. Visit http://www.dovepress.com/testimonials.php to read real quotes from published authors. 